\title{
Alterations in porcine intrahepatic sympathetic nerves after bisphenol A administration
}

\author{
Michael Thoene', Janusz Godlewski², Liliana Rytel ${ }^{3}$, Ewa Dzika ${ }^{1}$, \\ Ewa Bejer-Olenska ${ }^{4}$, Joanna Wojtkiewicz ${ }^{4,5}$
}

${ }^{1}$ Department of Medical Biology, Faculty of Health Sciences, University of Warmia and Mazury in Olsztyn, Poland

${ }^{2}$ Department of Human Histology and Embryology, School of Medicine, Collegium Medicum, University of Warmia and Mazury in Olsztyn, Poland

${ }^{3}$ Department of Internal Medicine and Clinic, Faculty of Veterinary Medicine, University of Warmia and Mazury, Olsztyn, Poland

${ }^{4}$ Department of Pathophysiology, School of Medicine, Collegium Medicum, University of Warmia and Mazury, Olsztyn, Poland

${ }^{5}$ Laboratory of Regenerative Medicine. University of Warmia and Mazury in Olsztyn, Poland

\begin{abstract}
Introduction. Bisphenol A (BPA) is used in the chemical industry for manufacturing plastics which are used as food packaging. Data indicate that BPA is released from such products and is widely present in the environment and the human body. So far, the EFSA and the US FDA have determined "safe" BPA oral exposure levels, and a large amount of data indicates that BPA is harmful even at low-doses. Our previously performed analyses concerning BPA exposure demonstrated the impact of this substance on parasympathetic and peptidergic nerve fibers present within the liver. Therefore, this study concerns BPA exposure and sympathetic intrahepatic innervation in reference to several neuropeptides which modulate neuronal responses: cocaine and amphetamine regulated transcript (CART), galanin (GAL), calcitonin gene-regulated peptide (CGRP) and substance P (SP). Materials and methods. Fifteen young swine at 8 weeks of age were used as experimental models of the juvenile human liver. The pigs were divided into 3 groups and received capsules orally with bisphenol at a dose of 0.05 $\mathrm{mg} / \mathrm{kg}$ b.w./day; a dose of $0.5 \mathrm{mg} / \mathrm{kg}$ b.w./day and placebo capsules as a control. After 28 days of oral BPA intake, the animals were euthanized, perfused with $4 \%$ paraformaldehyde (PFA), and livers were collected and fixed in PFA. The cryostat sections were subjected to a routine double-labeling immunofluorescence technique. The primary antibodies were directed against dopamine beta hydroxylase $(\mathrm{D} \beta \mathrm{H})$, which is a marker for sympathetic nerves, and one of the investigated neuropeptides: CART, GAL, CGRP and SP, which co-localized the investigated nerves. Immunoreactive nerves were counted in the liver and the percentage presence of each neuronal combination in particular samples of each experimental group were determined and analyzed statistically.

Results. The BPA oral intake at low and ten times higher dosage caused an increase of the number of sympathetic nerve fibers within the porcine liver by $48.6 \%$ and $63.7 \%$, respectively. Moreover, BPA exposure caused an increased presence of sympathetic nerve fibers in these two experimental groups, which were co-localized with CART and GAL up to $65.9 \% / 173.2 \%$ and $147.4 \% / 126.3 \%$, respectively. At the lower BPA doses of $50 \mu \mathrm{g} / \mathrm{kg}$ b.w./day, the percentages of $\mathrm{SP}+/ \mathrm{D} \beta \mathrm{H}+$ and $\mathrm{CGRP}+/ \mathrm{D} \beta \mathrm{H}+$ nerve fibers were similar to the control.
\end{abstract}

\footnotetext{
Correspondence address: $M$. Thoene

Department of Medical Biology,

Faculty of Health Sciences,

University of Warmia and Mazury in Olsztyn

Żołnierska 14C str., 10-561 Olsztyn, Poland

e-mail: michael.thoene@uwm.edu.pl
} 
However at a ten times higher dose, BPA caused an increased number of $\mathrm{SP}+/ \mathrm{D} \beta \mathrm{H}+$ and $\mathrm{CGRP}+/ \mathrm{D} \beta \mathrm{H}+$ nerve fibers in the liver, up to $46.4 \%$ and $73.5 \%$ respectively.

Conclusions. BPA caused an increase in the number of sympathetic nerve fibers as well as sympathetic nerve fibers which co-localized with neuropeptides in the porcine liver. The increase in CART and GAL were exceptionally high even at low BPA doses. BPA food contamination may dysregulate liver sympathetic innervation, and thereby may change the oxygenated blood supply, alter metabolism and disrupt the activity of hepatic parenchymal cells. (Folia Histochemica et Cytobiologica 2018, Vol. 56, No. 2, 113-121)

Key words: BPA; intrahepatic innervation; sympathetic nervous system; D $\beta \mathrm{H}$; CART; GAL; CGRP; SP

\section{Introduction}

Bisphenol A (BPA) is an organic chemical compound with applications in the chemical industry for manufacturing many types of plastic which are mostly used as food packaging: household food containers, plastic water bottles and the lining of aluminum beverage cans [1]. A large amount of data indicates that BPA is released from plastic and polymer products because it is widely found in the environment: air, soil and water. Moreover BPA is also present in food, especially in cans, which most likely causes food exposure to BPA or its metabolites. This exposure has been reported in human serum, milk, urine and feces $[2,3]$.

BPA is considered to be an endocrine disrupting compound (EDC) because at the cellular level it acts as an agonist of $\alpha$ and $\beta$ subtype estrogen receptors. It is significantly (1000-2000-fold) weaker than $17 \beta$ estradiol, but acts similarly to estrogens causing a migration of the cytoplasmic receptor domain and activation of the estrogen nuclear receptor [4]. Plastic or polymer products with a BPA component were originally deemed to be safe, since they exhibit low concentrations of BPA. The European Food Safety Authority as well as the US Food and Drug Administration have determined BPA safe oral exposure levels to be below $4 \mu \mathrm{g} / \mathrm{kg}$ body weight/day and 5000 $\mu \mathrm{g} / \mathrm{kg}$ body weight/day, respectively [5,6]. At the same time, the safety of the exposure level of this EDC has been extensively discussed and much data has shown that BPA is harmful even at low-doses $[7,8]$. Experimental studies performed so far concerning BPA and its effect on the nervous system have mostly demonstrated its impact as disrupted sexual differentiation and memory impairments, as well as changes in behavior such as hyperactivity and aggressiveness [9]. Moreover, a large amount of data has shown that BPA may not only change physiological processes in adults, but also could be destructive and cause alterations in nervous system development with accompanying morphological changes. In vitro, BPA significantly impairs the proliferation of neural progenitor cells and at high-doses it is even cytotoxic to them [10].
The in vivo experiments have demonstrated that BPA disrupts enteric innervation within particular parts of the porcine small intestine $[11,12]$.

Our previously performed analyses demonstrated the influence of BPA exposure on intrahepatic innervation components. Significant changes and increases in the number of parasympathetic and peptidergic nerve fibers present within the liver have been correlated with high BPA dosage levels [13, 14]. To complete the series of experimental studies concerning BPA and intrahepatic innervation, we decided to assess the potential changes of the sympathetic part of the autonomic nervous system with reference to several neuropeptides which modulate the neuronal response: cocaine- and amphetamine-regulated transcript (CART), galanin (GAL), calcitonin gene-regulated peptide (CGRP) and substance P (SP).

The assumption was made that experimental oral BPA exposure is similar to food BPA intake and could simulate BPA contamination in children and adolescents. The study describes the changes of intrahepatic innervation, because the small intestine digests food material and absorbs molecules which are transported directly to the liver through the hepatic portal vein.

As in previous analyses, we used young swine at the age of 8 weeks as a potential model of the juvenile human liver. The anatomical and physiological similarity between swine and human make the porcine liver one of the most useful experimental models for toxicological and pathophysiological studies $[15,16]$.

Using an innervated hepatic tissue to test ingested BPA exposure is reasonable since the liver is the first major organ exposed to dietary toxins. By testing alterations in the expression of nerve fibers in the liver, some suggestions concerning the impact of the above-mentioned alterations on liver function may be proposed. In this study we used an immunoflourescence technique to demonstrate the presence of dopamine beta hydroxylase $(\mathrm{D} \beta \mathrm{H})$, a marker specific for sympathetic nerve fibers, and four neuropeptides (CART, GAL, CGRP and SP) which can co-localize with $\mathrm{D} \beta \mathrm{H}$ in the liver. 
Table 1. Primary and secondary antibodies used in this study

\begin{tabular}{|c|c|c|c|c|}
\hline \multicolumn{5}{|c|}{ PRIMARY ANTIBODIES } \\
\hline Antisera & Code & Host species & Dilution & Supplier \\
\hline $\mathbf{D} \beta \mathbf{H}$ & MAB 308 & mouse & $1: 1000$ & Chemicon International Inc, UK; www.chemicon.com \\
\hline CART & H-003-61 & rabbit & $1: 22000$ & $\begin{array}{l}\text { Phoenix Europe } \\
\text { www.phoenixpeptide.com }\end{array}$ \\
\hline GAL & RIN7153 & rabbit & 1:10000 & Peninsula Labs, US; see Bachem AG; www.bachem.com \\
\hline CGRP & 11189 & rabbit & 1:10000 & MP Biomedicals; www.mpbio.com \\
\hline SP & $8450-0505$ & rabbit & 1:10000 & Biogenesis Inc, www.biogenesis.co.uk \\
\hline \multicolumn{5}{|c|}{ SECONDARY ANTIBODIES } \\
\hline & & & Dilution & Supplier \\
\hline \multicolumn{3}{|c|}{$\begin{array}{l}\text { Donkey anti-mouse } \operatorname{IgG}(\mathrm{H}+\mathrm{L}) \text { conjugated } \\
\text { with FITC }\end{array}$} & 1:800 & 715-095-151; Jackson IR Lab, US; ww.jacksonimmuno.com \\
\hline \multicolumn{3}{|c|}{ Biotinylated goat anti-rabbit immunoglobulins } & 1:1000 & E0432, DAKO Corporation, US, www.dakousa.com \\
\hline \multicolumn{3}{|c|}{$\begin{array}{l}\text { Biotin conjugated } \mathrm{F}(\mathrm{ab}) \text { ' fragment of affinity } \\
\text { Purified anti-rabbit } \operatorname{IgG}(\mathrm{H}+\mathrm{L})\end{array}$} & 1:1000 & 711-1622, BioTrend, Germany; www.biotrend.com \\
\hline \multicolumn{3}{|c|}{ CY3- conjugated Streptavidin } & 1:9000 & 016-160-084, Jackson IR Lab, US; www.jacksonimmuno.com \\
\hline
\end{tabular}

Abbreviations: CART — cocaine- and amphetamine-regulated transcript; CGRP — calcitonin gene- regulated peptide; $\mathrm{D} \beta \mathrm{H}$ - dopamine beta-hydroxylase; FITC — fluorescein isothiocyanate; GAL — galanin; SP — substance P. CY3- conjugated Streptavidin was used for detecting biotinylated antibodies (Cy3 excitation at $550 \mathrm{~nm}$ induces maximal emission at $570 \mathrm{~nm}$ ).

\section{Materials and methods}

Animals and design of experiment. The study was performed on 15 Large White Polish juvenile female pigs at the initial age of 8 weeks and about 18-20 kg body weight (b.w.). Pigs were kept under typical laboratory conditions adapted for this animal species at the Faculty of Veterinary Medicine, University of Warmia and Mazury in Olsztyn (Poland). The experiment was performed according to the instructions of the Local Ethical Committee for Experiments on Animals in Olsztyn, decision number (28/2013).

After a 3-day adaptive period, the pigs were randomly divided into three experimental groups and received bisphenol A (BPA) (cat. no. 239658, SigmaAldrich, St. Louis, MO, USA) or placebo in gelatin capsules for 28 days of feeding. 1) Control group - placebo (empty gelatin capsules);

2) Experimental group I - capsules with BPA at a dose of $0.05 \mathrm{mg} / \mathrm{kg}$ b.w./day;

3) Experimental group II - capsules with BPA at a dose 10 times higher $-0.5 \mathrm{mg} / \mathrm{kg}$ b.w./day.

All animals were weighed in order to determine their body weight and calculate the proper dosage of BPA every four days before the morning feeding.

Tissue sampling. After 28 days of oral BPA intake, the animals were premedicated with azaperone (Stressnil, Janssen Pharmaceutica, Bruxelles, Belgium; $2.0 \mathrm{mg} / \mathrm{kg}$ body weight, i.m. ). After about $30 \mathrm{~min}$. the animals were euthanized using an overdose of sodium thiopental intravenously
(50 mg/kg b.w; Sandoz, Kundl-Rakúsko, Austria) and perfused transcardially with $4 \%$ buffered paraformaldehyde (PFA). Tissues were collected from all animals. Sections of liver tissue were fixed in the same PFA solution, rinsed in phosphate buffered saline (PBS, $\mathrm{pH}$ 7.4) for three days and kept in $18 \%$ sucrose at $4{ }^{\circ} \mathrm{C}$. After two weeks, the tissue samples were frozen at $-23^{\circ} \mathrm{C}$ and cut into $10 \mu \mathrm{m}$-thick sections using a microtome (Microm, HM 525, Walldorf, Germany).

Immunofluorescent labeling and histomorphometry. The sections were subjected to a routine double-labeling immunofluorescence technique according to the method described previously by Wojtkiewicz et al. [16]. In short, overnight incubation was performed at $37^{\circ} \mathrm{C}$ with a mixture of two primary antibodies directed towards the dopamine beta hydroxylase $(\mathrm{D} \beta \mathrm{H})$ marker for sympathetic nerves and one of the studied neuropeptides: CART, GAL, CGRP or SP. The specification of primary and secondary antibodies which were used in the present study is presented in Table 1. Afterwards, the samples were incubated for $1 \mathrm{~h}\left(37^{\circ} \mathrm{C}\right)$ with species-specific antisera conjugated to FITC or biotin, which was visualized by streptavidin-CY3.

During the present investigation, the standard controls for the specificity of "primary" antibodies were performed. These included pre-absorption of the particular antisera with appropriate antigens, as well as "omission" and "replacement" tests that completely eliminated immunofluorescent signals.

Initially, the determination of the average number of $\mathrm{D} \beta \mathrm{H}$-positive nerve fibers per microscopic field of view 
$\left(0.1 \mathrm{~mm}^{2}\right)$ was made. Nerve fibers were counted in five sections per animal (in five view fields per section). Subsequently, the number of $\mathrm{D} \beta \mathrm{H}-\mathrm{LI}$ nerve fibers which were immunoreactive with the other studied substances was counted. $300 \mathrm{D} \beta \mathrm{H}$ immunoreactive nerve fibers in particular sections from each animal were counted for the presence of co-localized neuropeptides, and these numbers were considered to represent $100 \%$ for each combination. This was performed in order to determine what percentages of $\mathrm{D} \beta \mathrm{H}$-positive nerve fibers were immunoreactive with CART, GAL, CGRP or SP.

The measurements of the number of immunoreactive nerve fibers were performed by two investigators. Double-labeled nerve fibers were visualized under an Olympus BX51 microscope (Olympus, Tokyo, Japan) equipped with epi-fluorescence and appropriate filter sets. To prevent double counting of the same nerve fibers, the evaluated sections were located at least $100 \mu \mathrm{m}$ apart.

Statistical analysis. The obtained results were pooled and presented as mean \pm SEM. Statistical analysis was performed using Student's t test (Graphpad Prism v. 6.0; GraphPad Software Inc., San Diego, CA, USA), and the differences were considered statistically significant at $\mathrm{p} \leq 0.05$.

\section{Results}

\section{$B P A$ caused an increase of sympathetic nerve density within the liver}

In comparison to the control group, there was a significant increase in the number of observed sympathetic nerves $(\mathrm{D} \beta \mathrm{H}+)$, analyzed in the viewfield area, for the group of animals which were fed a BPA oral exposure at $0.05 \mathrm{mg} / \mathrm{kg} \mathrm{b.w} / \mathrm{day}$, and the group which was fed $0.5 \mathrm{mg} / \mathrm{kg}$ b.w./day; $48.6 \%$ and $63.7 \%$ respectively (Table 2, Fig. 1).

\section{$B P A$ caused an increased presence of sympathetic nerves co-localized with the analyzed neuropeptides} $\mathrm{CART} / \mathrm{D} \beta \mathrm{H}$ immunoreactive nerve fibers, at an initial BPA oral exposure of $0.05 \mathrm{mg} / \mathrm{kg}$ b.w./day, were $65.9 \%$ more abundant than in the control, and statistically significant (Table 2, Fig. 1). At an exposure ten times higher, the $\mathrm{CART} / \mathrm{D} \beta \mathrm{H}$ immunoreactive nerve presence was $173.2 \%$ higher, in comparison to the control.

Furthermore at the low and high BPA dosage, the percentage of GAL/D $\beta \mathrm{H}$ immunoreactive nerve fibers was higher (147.4\% and $126.3 \%$, respectively) than in the control group (Table 2, Fig. 2).

At a BPA oral intake of $0.05 \mathrm{mg} / \mathrm{kg}$ b.w./day, the percentages of $\mathrm{SP}+/ \mathrm{D} \beta \mathrm{H}+$ and $\mathrm{CGRP}+/ \mathrm{D} \beta \mathrm{H}+$ nerve fibers were similar to the control. However at a ten times higher exposure level, the expression of $\mathrm{SP}+/ \mathrm{D} \beta \mathrm{H}+$ was $46.4 \%$ and $\mathrm{CGRP}+/ \mathrm{D} \beta \mathrm{H}+$ was $73.5 \%$ higher than in the control group (Table 2, Fig. 3,4 , statistically significant, $\mathrm{p} \leq 0.05$ ).
The results reported above are presented in Table 2 and representative images of the immunocytochemical co-localization of $\mathrm{D} \beta \mathrm{H}$ with the chosen neuronal markers are shown in Figures 1-4.

\section{Discussion}

Anatomical studies have described that porcine liver in the portal area and in the parenchyma is innervated by abundant nerve fibers of the autonomic nervous system [18]. In the portal triad, the sympathetic nerves innervate the interlobular branches of the hepatic artery, portal vein and the bile duct. In the hepatic parenchyma, the sympathetic nerve fibers run from the portal area, and their terminal is in close vicinity to hepatocytes as well as other cells located in this area [18]. Comparison among higher mammalian species such as pig, dog, monkey and human demonstrate a high anatomic resemblance in the hepatic innervation between them $[18,19]$.

The results of this study show an increase in the presence of $\mathrm{D} \beta \mathrm{H}$ immunoreactive nerve fibers and this is comparable to our previous analyses which have also demonstrated an overexpression of parasympathetic and peptidinergic nerves fibers within porcine liver after BPA oral intake [13,14]. Such results suggest that BPA food contamination may disrupt proper innervation and balance between main components of the autonomic nervous system within liver. It could be assumed that sympathetic nerve fiber overexpression may cause improper blood flow within the portal area due to potential constriction of the interlobular artery. Similarly, within hepatic parenchyma such an increase of innervation may impair blood supply within the sinusoidal area and also could have an impact on hepatocyte metabolism; the activity of Kupffer cells and hepatic stellate cells [20]. Sympathetic regulation within the parenchyma of hepatic lobules is not only involved with the regulation of liver physiology, but it could also be related with several pathophysiological processes such as liver repair and regeneration [21].

Our results also show an increase in $\mathrm{D} \beta \mathrm{H}$ nerves which were co-localized with SP and CGRP neuropeptides in response to BPA exposure in a dose related manner. It is known that SP- and CGRP-immunoreactive (IR) nerve fibers have an afferent course to the spinal sensory ganglia and have sensory function in the alimentary tract and its glands [22, 23]. Comparative anatomical studies concerning SP nerve fibers have been conducted in many species including human but not in porcine. These studies have demonstrated numerous SP immunoreactive nerve fibers, which are present around the interlobular veins and arteries, with a lack of such innervation around the interlobular 
Table 2. Neurochemical characteristics and changes in neuropeptide expression in porcine intrahepatic sympathetic nerves under physiological conditions and after bisphenol A (BPA) administration. Nerve fibers were counted in five sections per animal (in five view fields per section). *These results are statistically significant at $\mathrm{p} \leq 0.05$

\begin{tabular}{|l|l|l|l|}
\hline \multirow{2}{*}{ Neurochemical characteristic of fibers } & \multicolumn{3}{|c|}{ Experimental group } \\
\cline { 2 - 4 } & Control & Low-dose BPA & High-dose BPA \\
\hline $\mathrm{D} \beta \mathrm{H}^{+}$ & $21.2 \pm 2.08$ & $31.5 \pm 1.85^{*}$ & $34.7 \pm 1.61^{*}$ \\
\hline $\mathrm{D} \beta \mathrm{H}^{+} / \mathrm{CART}^{+}$ & $8.2 \pm 1.65$ & $13.6 \pm 1.57^{*}$ & $22.4 \pm 3.26^{*}$ \\
\hline $\mathrm{D} \beta \mathrm{H}^{+} / \mathrm{GAL}^{+}$ & $3.8 \pm 0.96$ & $9.4 \pm 2.01^{*}$ & $8.6 \pm 1.57^{*}$ \\
\hline $\mathrm{D} \beta \mathrm{H}^{+} / \mathrm{CGRP}^{+}$ & $9.8 \pm 1.59$ & $10.6 \pm 1.77$ & $17.0 \pm 2.81^{*}$ \\
\hline $\mathrm{D} \beta \mathrm{H}^{+} / \mathrm{SP}^{+}$ & $5.6 \pm 1.63$ & $6.2 \pm 1.88$ & $8.2 \pm 1.98^{*}$ \\
\hline
\end{tabular}

Abbreviations: BPA — bisphenol A; C — control animals; E1 — low-dose BPA group, $0.05 \mathrm{mg} / \mathrm{kg}$ b.w./day for 28 days); E2 — high dose BPA group $(0.5 \mathrm{mg} / \mathrm{kg}$ b.w./ day for 28 days $)$
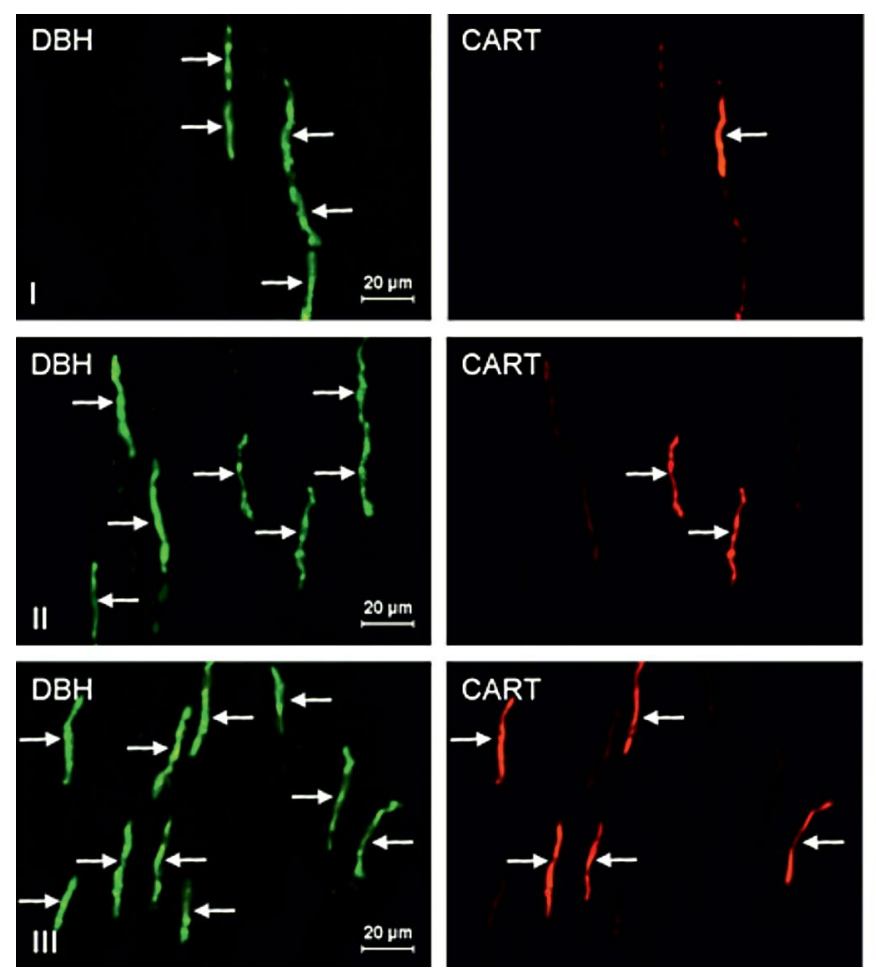
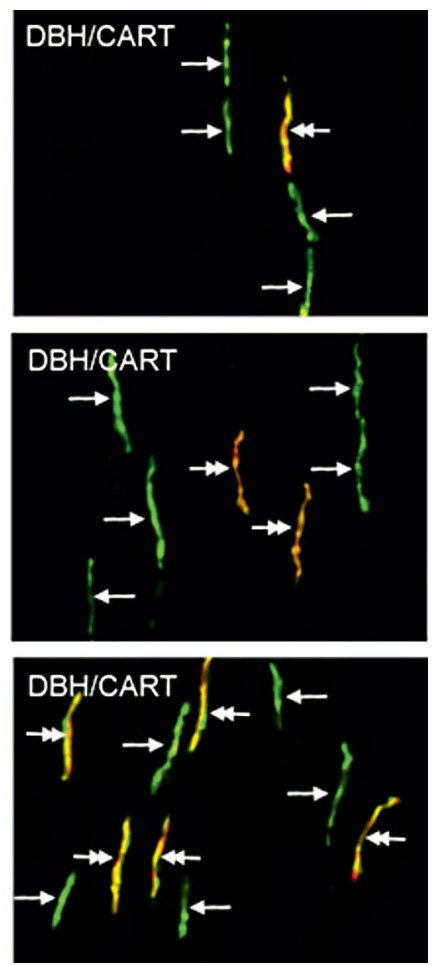

Figure 1. Representative images of immunofluorescent detection of $\mathrm{D} \beta \mathrm{H}$-, and CART-immunoreactive nerve fibers in the livers of control pigs (I) or pigs exposed to low (II) or high doses (III) of bisphenol A, as described in Methods. D $\beta \mathrm{H}$ - or CART-immunoreactive (IR) nerve fibers are indicated with arrows, $\mathrm{D} \beta \mathrm{H}$ - and CART-IR nerve fibers are indicated with double-headed arrows. Scale bars in all figures: $20 \mu \mathrm{m}$.

bile duct. Furthermore, SP-IR nerve fibers are also observed in the hepatic parenchyma. So far, these have been classified as peptidergic nerve fibers [19]. Our previous studies, as well as the study presented above, have shown that SP neuropeptide within the porcine liver is colocalized with parasympathetic and sympathetic nerve fibers as well as nerve fibers alone $[13,14]$ Other data have demonstrated that SP-IR nerves fibers could cause vasodilation and modulate microcirculation in hepatic sinusoids [20].
The CGRP-IR nerve fibers innervate the interlobular artery and bile duct; however, they are not present in the intralobular region of animal and human liver [19]. Other data concerned with skin innervation and internal microcirculation demonstrate that SP and CGRP are simultaneously released from sensory fibers and could cause short- and long-term vasodilation and plasma extravasation [24]. On the basis of the results of our study, it might be suspected that dose related BPA exposure changes the normal pattern of hepatic 

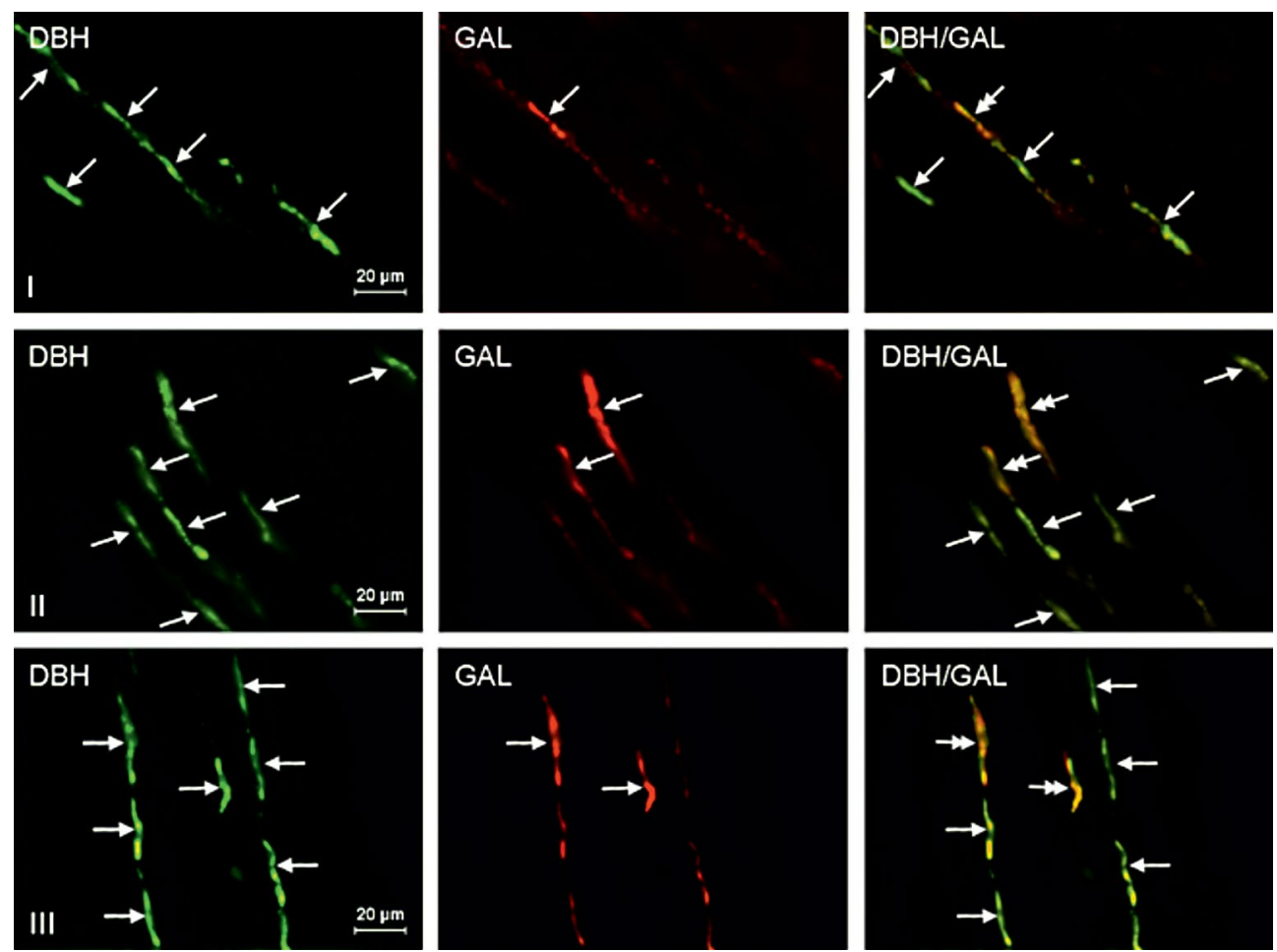

Figure 2. Representative images of immunofluorescent detection of $\mathrm{D} \beta \mathrm{H}$ - and GAL-immunoreactive nerve fibers in the livers of control pigs (I) or pigs exposed to low (II) or high doses (III) of bisphenol A, as described in Methods. D $\beta \mathrm{H}$ - or GAL-immunoreactive (IR) nerve fibers are indicated with arrows, $\mathrm{D} \beta \mathrm{H}$ - and GAL-IR nerve fibers are indicated with double-headed arrows. Scale bars in all figures: $20 \mu \mathrm{m}$.
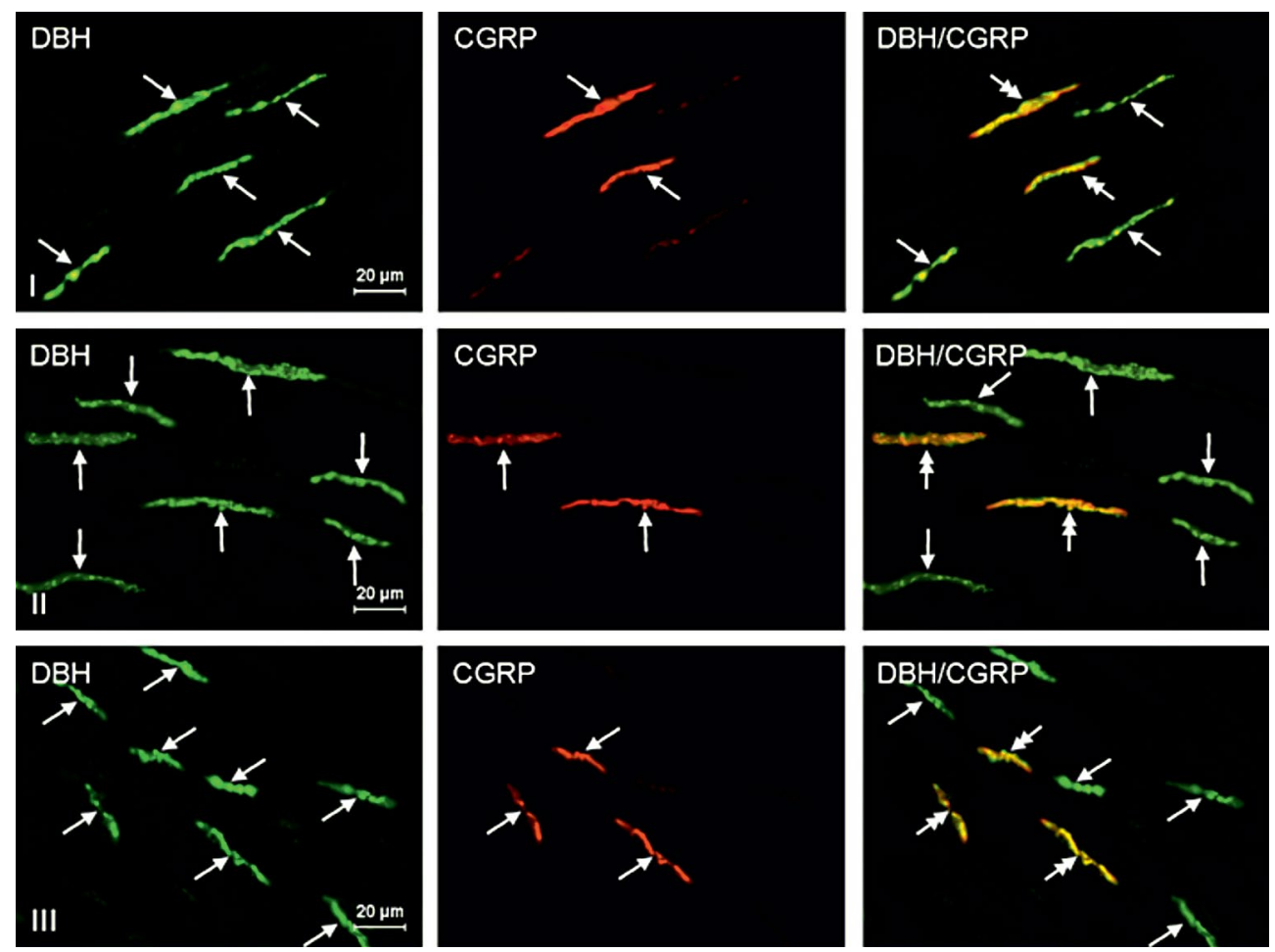

Figure 3. Representative images of immunofluorescent detection of $\mathrm{D} \beta \mathrm{H}$ - and CGRP-immunoreactive nerve fibers in the livers of control pigs (I) or pigs exposed to low (II) or high doses (III) of bisphenol A, as described in Methods. D $\beta \mathrm{H}$ - or CGRP-immunoreactive (IR) nerve fibers are indicated with arrows, $\mathrm{D} \beta \mathrm{H}$ - and CGRP-IR nerve fibers are indicated with double-headed arrows. Scale bars in all figures: $20 \mu \mathrm{m}$. 

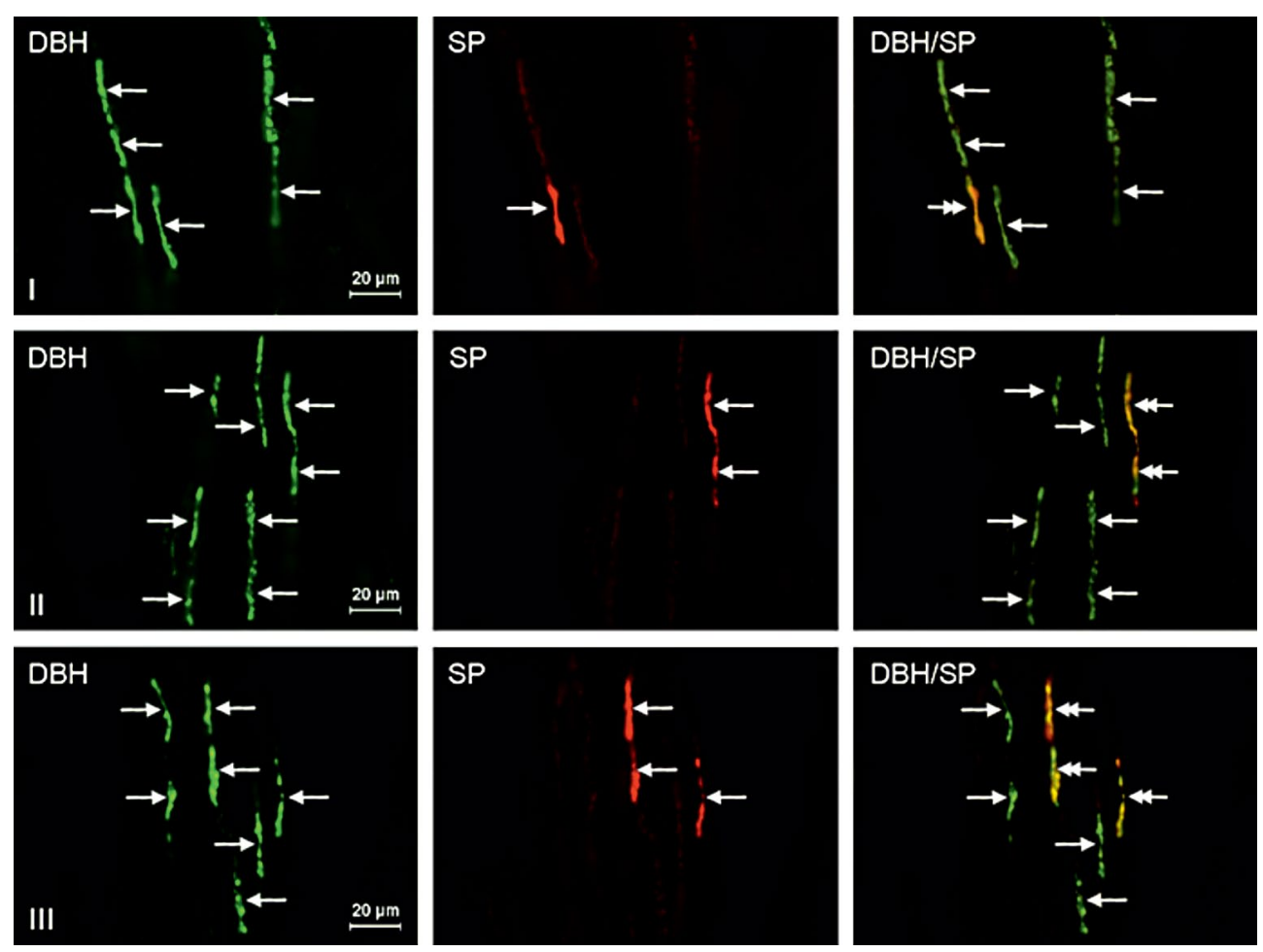

Figure 4. Representative images of immunofluorescent detection of $\mathrm{D} \beta \mathrm{H}$ - and SP-immunoreactive nerve fibers in the livers of control pigs (I) or pigs exposed to low (II) or high doses (III) of bisphenol A. as described in Methods. D $\beta$ H- or SP-immunoreactive (IR) nerve fibers are indicated with arrows, $\mathrm{D} \beta \mathrm{H}$ - and SP-IR nerve fibers are indicated with double-headed arrows. Scale bars in all figures: $20 \mu \mathrm{m}$.

innervation, which could disturb blood and bile flow in the portal area and microcirculation within hepatic sinusoids.

So far, BPA is considered to be an endocrine disrupting compound which reacts with nuclear estrogen receptor [4]. Moreover, it has also been described that BPA could react with membrane epithelial growth factor receptor (EGFR) and via ERK1/2 and AKT signaling pathways. This could activate cellular growth and cell proliferation; especially in cancer cells [2]. Nevertheless, there is a lack of experimental data which could directly demonstrate how BPA could impair the components of the peripheral nervous system. There is much data concerned with brain physiology and the impact of BPA exposure which causes disruptive patterns of behavior [9].

Another result of our study clearly demonstrates the presence of sympathetic nerves which are co-localized with galanin in porcine liver. The distribution of such nerves in this organ has not been clearly evidenced, so far. Our results show an increase in the density of immunoreactive $\mathrm{D} \beta \mathrm{H}^{+} / \mathrm{GAL}^{+}$nerve fibers, and this alteration was visible even at low BPA doses. Data from the literature indicate that current in-vitro studies are mostly concerned with different hormone dependent cells but not with hepatocytic cell cultures. These experiments demonstrate the impact of BPA on estrogen receptor up-regulation which causes cell activation and cell proliferation even at very low BPA concentrations [8]. Other data show galanin co-localization in sympathetic nerve fibers, but this was performed in dog liver. Most likely, galanin in these nerves acts as a neuromodulator and has a potential role in hepatic glucose release $[25,26]$.

The results of our study also demonstrate a significant increase in the presence of sympathetic nerve fibers which were co-localized with CART within the liver. Although CART is widely present within the enteric innervation of the alimentary tract, the exact function of this neuropeptide still seems to be unclear. It is possible that CART within the enteric neurons fulfils a neuroprotective role [27]. However, the widespread presence of CART in intestinal innervation more likely suggests that CART could be a modulator of motility and secretion within the alimentary tract $[28,29]$. It should be emphasized that CART co-expression within sympathetic nerve fibers in mammalian liver has been demonstrated for the first time. Furthermore, a distinct increase of CART immunoreactive nerve fibers caused by BPA 
exposure even at low doses is worth underlining. It is known that CART is not only a neurotransmitter in the central nervous or autonomic nervous system, but CART most likely plays a role as a hormone, because this presence has been documented in the endocrine cells of the adrenal medulla and the C-cells of the thyroid gland [30].

In summary, it should be highlighted that each neuropeptide that was studied demonstrated increased levels of expression within the sympathetic nerves of hepatic tissue. The increase of the density of CART- and GAL-IR sympathetic nerve fibers was high even at low BPA doses. $\mathrm{D} \beta \mathrm{H}$ expression was also increased, which indicates an over-activation of the sympathetic nervous system. Compiling the results of our studies with previously performed analyses concerning BPA exposure and the parasympathetic and peptidinergic parts of liver innervation [13, 14]; we come to the conclusion that BPA causes a general dysregulation of nerve fibers. This evidence indicates that BPA exposure even at recommended safe levels is still far too high. Furthermore, it is hoped that this study may be an early step in the determination of linking BPA exposure to pathological disease states.

\section{Acknowledgements}

This study was supported by statutory grant No. 25.610.001-300, Faculty of Medical Sciences, the University of Warmia and Mazury in Olsztyn, Poland.

The authors wish to thank Pawel Golawski and Paweł Wojtacha for their skillful technical assistance.

\section{References}

1. Tsai WT. Human health risk on environmental exposure to Bisphenol-A: a review. J Environ Sci Health C Environ Carcinog Ecotoxicol Rev. 2006; 24(2): 225-255, doi: 10.1080/10590500600936482, indexed in Pubmed: 17114111.

2. Murata M, Kang JH. Bisphenol A (BPA) and cell signaling pathways. Biotechnol Adv. 2018; 36(1): 311-327, doi: 10.1016/j.biotechadv.2017.12.002, indexed in Pubmed: 29229539.

3. Vandenberg LN, Hauser R, Marcus M, et al. Human exposure to bisphenol A (BPA). Reprod Toxicol. 2007; 24(2): 139-177, doi: 10.1016/j.reprotox.2007.07.010, indexed in $\mathrm{Pu}-$ bmed: 17825522 .

4. Acconcia F, Pallottini V, Marino M. Molecular Mechanisms of Action of BPA. Dose Response. 2015; 13(4): 1559325815610582, doi: $10.1177 / 1559325815610582$, indexed in Pubmed: 26740804.

5. European Food Safety Authority (EFSA) Panel on Food Contact Materials, Enzymes, Flavourings and Processing Aids. Scientific Opinion on the risks to public health related to the presence of bisphenol A (BPA) in foodstuffs: Executive summary. . ; 13(1): . https://. EFSA Journal 2015; 13(1): 3978 https://efsa onlinelibrary wiley com/doi/epdf/10 2903/j efsa 20153978 Accessed. ; 14: April, doi: 10.2903/j.efsa.2015.3978.
6. U.S. Food and Drug Administration. Bisphenol A (BPA). Final report for the review of literature and data on BPA 6/6/2014. https://www fda gov/Food/IngredientsPackagingLabeling/FoodAdditivesIngredients/ucm166145 htm Accessed.; 14: April.

7. Vandenberg LN, Colborn T, Hayes TB, et al. Hormones and endocrine-disrupting chemicals: low-dose effects and nonmonotonic dose responses. Endocr Rev. 2012; 33(3): 378-455, doi: 10.1210/er.2011-1050, indexed in Pubmed: 22419778.

8. Welshons WV, Thayer KA, Judy BM, et al. Large effects from small exposures. I. Mechanisms for endocrine-disrupting chemicals with estrogenic activity. Environ Health Perspect. 2003; 111(8): 994-1006, indexed in Pubmed: 12826473.

9. Inadera H. Neurological Effects of Bisphenol A and its Analogues. Int J Med Sci. 2015; 12(12): 926-936, doi: 10.7150/ /ijms.13267, indexed in Pubmed: 26664253.

10. Kim K, Son TG, Kim SoJ, et al. Suppressive effects of bisphenol A on the proliferation of neural progenitor cells. J Toxicol Environ Health A. 2007; 70(15-16): 1288-1295, doi: 10.1080/15287390701434216, indexed in Pubmed: 17654246.

11. Szymanska K, Gonkowski S. Bisphenol A-Induced changes in the enteric nervous system of the porcine duodenum. Neurotoxicology. 2018; 66: 78-86, doi: 10.1016/j.neuro.2018.03.008, indexed in Pubmed: 29577965.

12. Szymanska K, Makowska K, Gonkowski S. The Influence of High and Low Doses of Bisphenol A (BPA) on the Enteric Nervous System of the Porcine Ileum. Int J Mol Sci. 2018; 19(3), doi: 10.3390/ijms19030917, indexed in Pubmed: 29558425.

13. Thoene M, Rytel L, Dzika E, et al. Bisphenol A Causes Liver Damage and Selectively Alters the Neurochemical Coding of Intrahepatic Parasympathetic Nerves in Juvenile Porcine Models under Physiological Conditions. Int J Mol Sci. 2017; 18(12), doi: 10.3390/ijms18122726, indexed in $\mathrm{Pu}-$ bmed: 29244763 .

14. Thoene MA, Rytel L, Dzika E, et al. Immunohistochemical characteristics of porcine intrahepatic nerves under physiological conditions and after Bisphenol A administration. Folia Morphol (Warsz). 2018 [Epub ahead of print], doi: 10.5603/ /FM.a2018.0027, indexed in Pubmed: 29569701.

15. Helke KL, Swindle MM. Animal models of toxicology testing: the role of pigs. Expert Opin Drug Metab Toxicol. 2013; 9(2): 127-139, doi: 10.1517/17425255.2013.739607, indexed in Pubmed: 23216131.

16. Nykonenko A, Vávra P, Zonča P. Anatomic Peculiarities of Pig and Human Liver. Exp Clin Transplant. 2017; 15(1): 21-26, indexed in Pubmed: 28122484.

17. Wojtkiewicz J, Rytel L, Makowska K, et al. Co-localization of zinc transporter 3 (ZnT3) with sensory neuromediators and/or neuromodulators in the enteric nervous system of the porcine esophagus. Biometals. 2017; 30(3): 393-403, doi: 10.1007/s10534-017-0014-1, indexed in Pubmed: 28417221.

18. Lin YS, Nosaka S, Amakata Y, et al. Comparative study of the mammalian liver innervation: an immunohistochemical study of protein gene product 9.5, dopamine beta-hydroxylase and tyrosine hydroxylase. Comp Biochem Physiol A Physiol. 1995; 110(4): 289-298, indexed in Pubmed: 7735898.

19. Akiyoshi H, Gonda T, Terada T. A comparative histochemical and immunohistochemical study of aminergic, cholinergic and peptidergic innervation in rat, hamster, guinea pig, dog and human livers. Liver. 1998; 18(5): 352-359, indexed in Pubmed: 9831365.

20. Streba LA, Vere CC, Ionescu AG, et al. Role of intrahepatic innervation in regulating the activity of liver cells. World 
J Hepatol. 2014; 6(3): 137-143, doi: 10.4254/wjh.v6.i3.137, indexed in Pubmed: 24672643.

21. Jensen KJ, Alpini G, Glaser S. Hepatic nervous system and neurobiology of the liver. Compr Physiol. 2013; 3(2): 655-665, doi: 10.1002/cphy.c120018, indexed in Pubmed: 23720325.

22. Barja F, Mathison R. Sensory innervation of the rat portal vein and the hepatic artery. J Auton Nerv Syst. 1984; 10(2): 117-125, indexed in Pubmed: 6205041.

23. Berthoud HR. Anatomy and function of sensory hepatic nerves. Anat Rec A Discov Mol Cell Evol Biol. 2004; 280(1): 827-835, doi: 10.1002/ar.a.20088, indexed in Pubmed: 15382018.

24. Schlereth T, Schukraft J, Krämer-Best HH, et al. Interaction of calcitonin gene related peptide (CGRP) and substance $\mathrm{P}$ (SP) in human skin. Neuropeptides. 2016; 59: 57-62, doi: 10.1016/j.npep.2016.06.001, indexed in Pubmed: 27344069.

25. Kowalyk S, Veith R, Boyle M, et al. Liver releases galanin during sympathetic nerve stimulation. Am J Physiol. 1992; 262(5 Pt 1): E671-E678, doi: 10.1152/ajpendo.1992.262.5.E671, indexed in Pubmed: 1375437.

26. Taborsky GJ, Dunning BE, Havel PJ, et al. The canine sympathetic neuropeptide galanin: a neurotransmitter in pan- creas, a neuromodulator in liver. Horm Metab Res. 1999; 31(5): 351-354, doi: 10.1055/s-2007-978752, indexed in Pubmed: 10422734 .

27. Ekblad E. CART in the enteric nervous system. Peptides. 2006; 27(8): 2024-2030, doi: 10.1016/j.peptides.2005.12.015, indexed in Pubmed: 16759747.

28. Palus K, Rytel L. Co-localisation of cocaine- and amphetamine-regulated transcript peptide and vasoactive intestinal polypeptide in the myenteric plexus of the porcine transverse colon. Folia Morphologica. 2013; 72(4): 328-332, doi: 10.5603/fm.2013.0055.

29. Wojtkiewicz J, Gonkowski S, Bladowski M, et al. Characterisation of cocaine- and amphetamine- regulated transcript-like immunoreactive (CART-LI) enteric neurons in the porcine small intestine. Acta Vet Hung. 2012; 60(3): 371-381, doi: 10.1556/AVet.2012.032, indexed in Pubmed: 22903082.

30. Wierup N, Gunnarsdóttir A, Ekblad E, et al. Characterisation of CART-containing neurons and cells in the porcine pancreas, gastro-intestinal tract, adrenal and thyroid glands. BMC Neurosci. 2007; 8: 51, doi: 10.1186/1471-2202-8-51, indexed in Pubmed: 17625001. 
